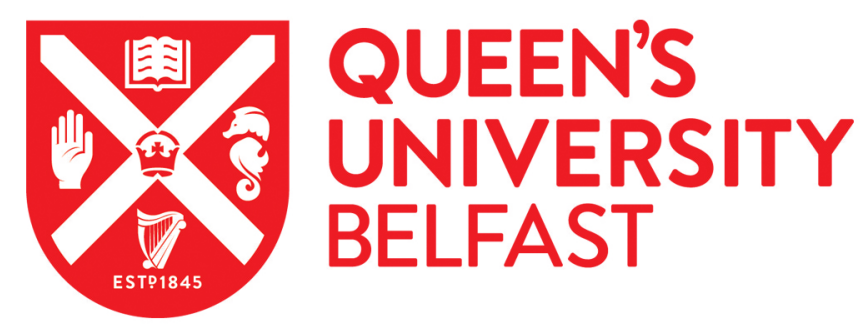

\title{
The development of an inline Raman spectroscopic analysis method as a quality control tool for hot melt extruded ramipril fixed-dose combination products
}

Andrews, G. P., Jones, D. S., Senta-Loys, Z., Almajaan, A., Li, S., Chevallier, O., Elliott, C., Healy, A-M., Kelleher, J., Madi, A., Gilvary, G. C., \& Tian, Y. (2019). The development of an inline Raman spectroscopic analysis method as a quality control tool for hot melt extruded ramipril fixed-dose combination products. International Journal of Pharmaceutics, 566, 476-487. https://doi.org/10.1016/j.ijpharm.2019.05.029

Published in:

International Journal of Pharmaceutics

Document Version:

Peer reviewed version

Queen's University Belfast - Research Portal:

Link to publication record in Queen's University Belfast Research Portal

Publisher rights

Copyright 2019 Elsevier.

This manuscript is distributed under a Creative Commons Attribution-NonCommercial-NoDerivs License

(https://creativecommons.org/licenses/by-nc-nd/4.0/), which permits distribution and reproduction for non-commercial purposes, provided the author and source are cited.

\section{General rights}

Copyright for the publications made accessible via the Queen's University Belfast Research Portal is retained by the author(s) and / or other copyright owners and it is a condition of accessing these publications that users recognise and abide by the legal requirements associated with these rights.

\section{Take down policy}

The Research Portal is Queen's institutional repository that provides access to Queen's research output. Every effort has been made to ensure that content in the Research Portal does not infringe any person's rights, or applicable UK laws. If you discover content in the

Research Portal that you believe breaches copyright or violates any law, please contact openaccess@qub.ac.uk. 


\section{The Development of an Inline Raman Spectroscopic Analysis Method}

2 as a Quality Control Tool for Hot Melt Extruded Ramipril Fixed-dose

3 Combination Products

4

5 Y. Tian ${ }^{1}$, Z. Senta-Loys ${ }^{1}$, A. Almajaan ${ }^{1}$, S. Li ${ }^{1}$, D.S. Jones ${ }^{1}$, O. Chevallier ${ }^{3}$, C. Elliot ${ }^{3}$, A.M.

6 Healy $^{2}$, J. F. Kelleher., A. M. Madi., G.C. Gilvary., G.P. Andrews ${ }^{1^{*}}$

7

$8{ }^{1}$ Pharmaceutical Engineering Group, School of Pharmacy, Queen's University Belfast, Belfast,

9 Northern Ireland, United Kingdom

$10 \quad 2$ School of Pharmacy and Pharmaceutical Sciences, Trinity College Dublin, Dublin, Ireland

$11{ }^{3}$ Institute for Global Food Security (IGFS), School of Biological Sciences, Queen's University

12 Belfast, Northern Ireland, United Kingdom

13

14 *corresponding author: Gavin P. Andrews, Pharmaceutical Engineering Group, School of

15 Pharmacy, 97 Lisburn Road, Belfast, United Kingdom, BT9 7BL.

Tel: +442890972646

Email:g.andrews@qub.ac.uk

18 Key words: hot melt extrusion; continuous manufacturing; process analytical technology; inline

19 Raman spectroscopy; multivariate data analysis; fixed-dose combination; anti-hypertension. 
Currently in the pharmaceutical industry, continuous manufacturing is an area of significant interest. In particular, hot-melt extrusion (HME) offers many advantages and has been shown to significantly reduce the number of processing steps relative to a conventional product manufacturing line. To control product quality during HME without process interruption, integration of inline analytical technology is critical. Vibrational spectroscopy (Raman, NIR and FT-IR) is often employed and used for real-time measurements because of the non-destructive and rapid nature of these analytical techniques. However, the establishment of reliable Process Analytical Technology (PAT) tools for HME of thermolabile drugs is challenging. Indeed, Raman effect is inherently weak and might be subject to interference such as scattering, absorption and fluorescence. Moreover, during HME, heating and photodecomposition can occur and disrupt spectra acquisition. The aim of this research article was to explore the use of inline Raman spectroscopy to characterise a thermolabile drug, ramipril (RMP), during continuous HME processing. Offline measurements by HPLC, LC-MS and Raman spectroscopy were used to characterise RMP and its main degradation product, ramipril-diketopiperazine (RMP-DKP, impurity K). A set of HME experiments together with inline Raman spectroscopic analysis were performed. The feasibility of implementing inline Raman spectroscopic analysis to quantify the level of RMP and RMP-DKP in the extrudate was addressed. Two regions in the Raman spectrum were selected to differentiate RMP and RMP-DKP. When regions were combined, a principle component analysis (PCA) model defined by these two main components (PC $1=50.1 \%$ and PC $2=45 \%$ ) was established. Using HPLC analyses, we were able to confirm that the PC 1 score was attributed to the level of RMP-DKP, and the PC 2 score was related to the RMP drug content. Investigation of the PCA scatterplot indicated that HME processing temperature was not the only

44 factor causing RMP degradation. Additionally, the plasticiser content, feeding speed and screw rotating speed can all contribute to the RMP degradation during HME processing. 


\section{Introduction}

47 The pharmaceutical industry has a well-established quality by testing regulatory framework for safeguarding the manufacture of both small molecule and biopharmaceutical products. This conservative process involves rigorous checks to ensure the quality of the final product and ultimately that it delivers the desired therapeutic efficacy. However, traditional manufacturing platforms and batch-based testing operations present inherent limitations for the commercialisation of modern complex drug delivery systems (Rantanen and Khinast, 2015). The implementation of innovative continuous manufacturing platforms provide process intensification and integration, coupled with real-time quality assurance tools; these developments offer significant potential to reduce the costs associated with traditional pharmaceutical and biopharmaceutical products (Badman and Trout, 2014). Furthermore, certain platforms that enable the use of true continuous manufacturing for "end-to-end" production of complex drug delivery systems may present a much-needed paradigm shift for both research development and commercial scale manufacturing. Additionally, traditional multistep processes may be naturally integrated with homogeneous processes such as extrusion and spray drying. With a thorough understanding of product processes, tailored complex formulations may be carefully engineered based upon a fundamental understanding of selected manufacturing platforms.

With the potential to revolutionise manufacturing processing and drug product quality and complexity, it is not surprising that there has been interest in techniques such as hot-melt extrusion (HME) over the last three decades (Repka et al., 2007). This growing interest in HME may be attributed to the wide range of possibilities in the production of pharmaceutical intermediates (e.g. crystalline, amorphous, cocrystalline and nanoparticles) and final dosage forms, (powder, tablet, granule, film, implant and vaginal ring) for different routes of administration, with tailored drug release profiles (e.g. controlled release, solubility enhancement) (Kelly et al., 2012; Li et al., 2016; Paradkar et al., 2010; Patil et al., 2016; Tian et al., 2018). HME also offers a continuous manufacturing platform without the use of solvent, 
which is environmental friendly, easy to scale-up and demonstrates good reproducibility.

Furthermore, a key aspect of continuous manufacturing is monitoring of process parameters

such as screw speed, feed rate, temperature and the potential to implement Process Analytical

Technologies (PAT) that permit the management of various Critical Quality Attributes (CQAs) throughout the process (Lang et al., 2014). Introduced by the Food and Drug Administration (FDA) in 2004, the adoption of PAT is part of a scientific risk-based framework intended to support innovation and efficiency in pharmaceutical development, manufacturing, and quality assurance (FDA, 2004). A PAT framework consists of designing and developing continuous monitoring and control strategies to understand and improve the CQAs of pharmaceutical products through inline, online or at-line measurements (Fonteyne et al., 2012; Laske et al., 2017; Wahl et al., 2013). It must be emphasised, however, the concepts and implementation of PAT and QbD framework is not new as process analysis and control have been widely adopted in many other industries (oil refinery, food, plastic and semiconductor). The application of PAT in pharmaceutical science and manufacturing, particularly in conjunction with the development of modern complex formulation and adoption of novel continuous manufacturing platforms is an exciting and challenging area in pharmaceutical manufacturing. Inline vibrational spectroscopic techniques are mainly used as PAT tools to characterise CQAs during continuous manufacturing because of the non-invasive and rapid nature of these methods (De Beer et al., 2011; Netchacovitch et al., 2015). FT-IR and Raman spectroscopy permit materials to be monitored during processing without the sample being removed from the process stream (Laske et al., 2017). Interestingly, when introducing PAT to a HME line, the effects of thermal and mechanical energy input on the integrity of drug may also be investigated. It offers the potential to generate an understanding of the mechanisms associated within extrusion, rather than the traditional 'black-box' treatment of this new pharmaceutical manufacturing technique (Eitzlmayr et al., 2014). Excess thermal and/or mechanical energy input can introduce unwanted changes to the pharmaceutical formulation, particularly when thermally labile drugs and/or excipients are 
ability to capture process induced degradation using PAT within HME can provide significant

101 advantages to the design and understanding of materials and process.

102 In our previous work, we reported a comparison between spray drying and hot-melt extrusion

103 for the continuous production of fixed-dose combination formulations for the treatment of 104 hypertension (Kelleher et al., 2018). Traditionally, batch manufacturing is utilized to produce 105 FDCs. In such setups, mixtures and intermediate products are going from one container to the 106 next, they are normally tested off-line and stored before being transferred to the next 107 processing step. These multi-batch disconnected processes normally result an extended lead 108 time before the final dosage form can be released. In continuous manufacturing processes, 109 such as spray drying (SD) and hot melt extrusion (HME), there is a continuous feed input and 110 product output in a one-step process. Inline process analytical techniques (PAT) framework 111 ensure desired critical quality attributes of the formulation are assessed and maintained 112 throughout the process, allowing real time manufacture, quality control and product relase 113 (Lee, 2015). With the potential benefts it may offer on product quality, manufacturing agility 114 and flexibility and cost of production, global pharmaceutical continous manufacturing market 115 is gaining moment reaching 1.9 Billion in 2017 with CAGR at 8.4\% (ZION market research, 116 2018).

117 Ramipril (RMP), a commonly prescribed angiotensin converting enzyme inhibitor was chosen as 118 the model drug. RMP is often prescribed together with other drugs such as hydrochlorothiazide 119 (HCTZ) to form a fixed dose combination (FDC) product with enhanced therapeutic efficacy. 120 However, hot-melt extrusion was found to result in significant degradation of RMP in 121 comparison to solvent-based spray drying. In this work, we report, for the first time, the 122 development of inline Raman spectroscopic analysis in combination with multivariate data 123 analysis to monitor RMP drug content and RMP degradation product during HME processing. In 124 the literature, several studies have shown RMP instability at different pH values, and under 
125 oxidative or thermal stresses (Hanyšová et al., 2005). The main degradation product after the

126 application of heat stress is ramipril-diketopiperazine (RMP-DKP) (De diego et al., 2010). Due to

127 thermal sensitivity, RMP processing by HME represents a real challenge. The aim of this work

128 was to demonstrate the application of inline Raman spectroscopy as a PAT tool for the

129 manufacture of a FDC formulation containing RMP. The presence of RMP-DKP was first

130 highlighted and identified using a series of offline measurements (HPLC and LC-MS). The

131 correlation of these results with the inline Raman spectra collected during HME processing was

132 probed. Multivariate data analysis model (PCA) were then used to characterize RMP and its

133 degradation product during HME processing. Important HME process attributes were identified

134 for RMP based FDC formulations. Using a Quality by Design approach (QbD), the knowledge-

135 based PCA model was further applied to the inline monitoring and analysis of RMP-HCTZ FDC

136 manufactured using HME (European Medicines Agency, 2015). 


\section{Material and methods}

140 Ramipril (RMP) was purchased from Kemprotec (Carnforth, England). Eudragit EPO was

141 generously gifted from Evonik Industries (Essen, Germany). Triethyl citrate (TEC) was

142 purchased from Lancaster synthesis Ltd (County, Country).

\subsection{Experimental Design}

144 An initial scoping design of extrusion process parameters and formulation factors (Table 1) was

145 conducted, where high and low levels of each process parameter were chosen in order to gain 146 maximum responses for the degradation of RMP. To highlight the degradation of RMP, several 147 parameters were considered: RMP and TEC (plasticiser) concentrations, feed rate, screw speed 148 and temperature. A total of fifteen experiments were conducted in order to probe the influence 149 of these factors on the content of RMP and RMP-DKP.

Table 1: Overview of the composition and process conditions employed in HME studies

\begin{tabular}{|l|l|l|l|l|l|l|}
\hline & \multicolumn{3}{|c|}{ Formulation variables } & \multicolumn{3}{c|}{ Process variables } \\
\hline Ext & RMP $_{\text {theo }}$ (\%) & TEC (\%) & EPO (\%) & $\begin{array}{c}\text { Feed speed } \\
\text { (rpm) }\end{array}$ & $\begin{array}{c}\text { Screw speed } \\
\text { (rpm) }\end{array}$ & Temp. ( ${ }^{\circ}$ C) \\
\hline S1 & 15 & 0 & 85 & manual & 60 & 140 \\
\hline S2 & 15 & 0 & 85 & manual & 60 & 110 \\
\hline S3 & 5 & 10 & 85 & manual & 60 & 110 \\
\hline S4 & 10 & 10 & 80 & manual & 60 & 110 \\
\hline S5 & 15 & 10 & 75 & manual & 60 & 110 \\
\hline S6 & 5 & 5 & 90 & 7 & 60 & 110 \\
\hline S7 & 10 & 5 & 85 & 7 & 60 & 110 \\
\hline S8 & 15 & 5 & 80 & 9 & 60 & 110 \\
\hline S9 & 5 & 5 & 90 & 2 & 5 & 110 \\
\hline S10 & 10 & 7.5 & 82.5 & 15 & 50 & 110 \\
\hline S11 & 10 & 7.5 & 82.5 & 15 & 50 & 110 \\
\hline S12 & 15 & 10 & 75 & 25 & 90 & 110 \\
\hline S13 & 15 & 10 & 75 & 20 & 70 & 100 \\
\hline S14 & 15 & 10 & 75 & manual & 100 & 110 \\
\hline S15 & 15 & 10 & 75 & manual & 100 & 140 \\
\hline
\end{tabular}


153 Polymer/plasticizer and drug at defined ratios were premixed using a mortar and pestle then

154 fed into a $10 \mathrm{~mm}$ twin-screw co-rotating extruder (Rondol Technology Ltd, France) with a twin155 screw powder feeder (Rondol Technology Ltd, France) at various feeding speed or manually. 156 The extruder die was $2 \mathrm{~mm}$ in diameter and an in-house custom designed inline Raman probe 157 was used (Figure 1). The extrudates were pelletised using a VARICUT pelletiser 158 (ThermoScientific, Germany)

\subsection{Inline Raman Measurements and Principle Component Analysis}

160 Inline Raman Spectra were collected with a Raman Rxn1 spectrometer (Kaiser Optical Systems, 161 Ann Arbor, MI, USA). For inline measurements, a high temperature and pressure immersion 162 probe (RAMAN RXN ${ }^{\mathrm{TM}}$ Probe specifically designed for HME) was inserted into the die head with a 163 custom-made fitting (Figure 1). An Invictus NIR diode laser was employed, with a wavelength of $164785 \mathrm{~nm}$ (Kaiser Optical Systems). All inline Raman spectra were recorded with a resolution of 2 $165 \mathrm{~cm}^{-1}$ and an exposure time of 0.5 seconds using a laser power of $400 \mathrm{~mW}$. Spectra were collected 166 every 20 seconds during experimentation. The run-time for a typical experiment was 167 approximately 30 minutes, with a 10-minute stabilisation period. Data collection was automated 168 using iC Raman software (version 4.1, METTLER TOLEDO) after stabilisation. Raman spectra 169 were automatically transferred and analysed using SIMCA software (version 14.1, Umetrics,

170 Umeå, Sweden). Principal Component Analysis (PCA) was used to highlight principle 171 components of all the spectra collected during HME processing. To reduce random noise and 172 undesired perturbations in the signal particularly at high temperature, Standard Normalised 173 Variate (SNV) and Multiplicative Scatter Correction (MSC) pre-processing and Savitzky-Golay 174 (SG) smoothing were applied before PCA.

176 HPLC offline analysis was performed in order to quantify both RMP and RMP-DKP. Pelletised 177 extrudates $(25-30 \mathrm{mg}$ ) were dissolved in 0.1M HCL (representing gastric media) to obtain a 
concentration of RMP of $25 \mu \mathrm{g} / \mathrm{mL}$. After filtration through hydrophilic PTFE filters $(0.45 \mu \mathrm{m}$,

179 Fisher Scientific Ireland Ltd., Dublin, Ireland), samples were analysed with an Agilent 1260

180 Infinity Series HPLC (Agilent Technologies, Cheadle, UK). A Kinetex® C18 column (150 mm

181 length, diameter $4.6 \mathrm{~mm}$, particle size $5 \mu \mathrm{m}$ ) was used as the stationary phase. The mobile phase

182 consisted of an aqueous solution containing $0.1 \mathrm{M}$ sodium perchlorate adjusted with phosphoric

183 acid to pH 2.5 (mobile phase A), and acetonitrile (mobile phase B). The samples were injected

184 automatically with an auto-sampler $(10 \mu \mathrm{L})$, and the flow rate varied between 0.8 and 1.5

$185 \mathrm{ml} / \mathrm{min}$ as described in Table 2. Detection was performed using a UV detector at $210 \mathrm{~nm}$.

186 Table 2: Details of HPLC gradient elution method including time (min), mobile phase

187 composition and flow rate $(\mathrm{mL} / \mathrm{min})(\%)$

188

\begin{tabular}{|c|c|c|c|}
\hline Time (min) & Mobile Phase A (\%) & Mobile Phase B (\%) & Flow (mL/min) \\
\hline 0 & 70 & 30 & 0.8 \\
\hline 5 & 40 & 60 & 1.5 \\
\hline 6 & 70 & 30 & 1 \\
\hline 9 & 70 & 30 & 1 \\
\hline 10 & 70 & 30 & 0.8 \\
\hline
\end{tabular}

189 The specificities required by for RAM formulation are i) an assay level of $90-105 \%$ of the label

190 claim for RAM; ii) Levels of the major metabolite RAM-DKP of $\leq 5.0 \%$.(Angeli and Trezza, 2009)

191 Therefore, we have constructed a calibration curve with RMP concentration from 5 to $50 \mu \mathrm{g} / \mathrm{mL}$.

192 Linearity was observed with a goodness fit $\left(\mathrm{R}^{2}\right)$ of 0.999 . We also normallised the RMP

193 degradation product in respective to the stated RMP content. It was defined as the area ratio

194 between the RMP-DKP peak and the RMP reference peak expressed as equation 1. Pure RMP

195 peak area at a defined concentration of $25 \mu \mathrm{g} / \mathrm{mL}$ was used as internal standard.

196

$$
\text { Normalised } \% R M P-D K P=\frac{\operatorname{Area}(R A M-D K P)}{\operatorname{Area}\left(R A M_{\text {ref }}\right)} \times \% R A M_{\text {theo }} \quad \text { equation } 1
$$

197 The percentage of RMP-DKP was classified at three levels: 


\subsection{LC-MS Analysis}

LC-MS analysis was performed in order to identify RMP impurities after HME. To test for RMP degradation product (RMP-DKP), pure RMP was heated at two temperatures, $100^{\circ} \mathrm{C}$ and $140^{\circ} \mathrm{C}$, for two hours on a hotplate. Samples were then dissolved in simulated gastric media (0.1M HCL), filtered and analysed. Analyses were carried out on a Waters Acquity UPLC I-Class system (Milford, MA, USA) coupled to a Waters Xevo G2-XS QTof mass spectrometer (Manchester, UK) with an electrospray ionisation source operating in positive or negative mode with lock-spray interface for real time accurate mass correction. Instrument settings were as follows: source temperature was set at $120^{\circ} \mathrm{C}$, cone gas flow at $50 \mathrm{~L} / \mathrm{h}$, desolvation temperature at $450{ }^{\circ} \mathrm{C}$, and desolvation gas flow at $850 \mathrm{~L} / \mathrm{h}$. The capillary voltage was set at $1.0 \mathrm{kV}$ in positive mode and 0.5 $\mathrm{kV}$ in negative mode, respectively. Source offset was 80 (arbitrary unit). Mass spectra data were acquired in continuum mode using the MSE function (low energy: $4 \mathrm{eV}$; high energy: ramp from

21115 to $30 \mathrm{eV}$ ) over the range m/z 50-1200 with a scan time of $0.1 \mathrm{~s}$. A lock-mass solution of 212 Leucine Enkephalin $(1 \mathrm{ng} / \mu \mathrm{L})$ in methanol/water containing $0.1 \%$ formic acid $(1: 1, \mathrm{v} / \mathrm{v})$ was 213 continuously infused into the MS via the lock-spray at a flow rate of $10 \mu \mathrm{L} / \mathrm{min}$. The 214 chromatographic separation was conducted on a Waters Acquity UPLC HSS T3 column (100 mm $215 \times 2.1 \mathrm{~mm}, 1.8 \mu \mathrm{m})$ equipped with an Acquity UPLC HSS T3 VanGuard pre-column $(100 \AA ̊, 1.8 \mu \mathrm{m}$, $2162.1 \mathrm{~mm} \mathrm{X} 5 \mathrm{~mm}$ ). The column oven temperature was set at $45^{\circ} \mathrm{C}$, injection volume at $5 \mu \mathrm{L}$ and 217 flow rate at $0.4 \mathrm{~mL} / \mathrm{min}$. The mobile phase consisted of $(\mathrm{A})$ water with $0.1 \%$ formic acid and (B) 218 methanol with $0.1 \%$ formic acid. The gradient was set as follows: 2.00 min of $99 \%$ (A) followed 219 by a linear increase from 1 to $99 \%$ (B) over $16.00 \mathrm{~min}$, isocratic cleaning step at 99\% (B) for $2200.50 \mathrm{~min}$, then returned to initial conditions $99 \%$ (A) over $0.25 \mathrm{~min}$ and column equilibration 221 step at $99 \%(A)$ for $1.75 \mathrm{~min}$. 


\section{Results and discussion}

224

225

226

227

228

229

230

231

232

233

234

235

\subsection{HPLC and LC-MS analysis of RMP and its degradation product}

Inline Raman spectroscopic analysis and robustness of offline analytical techniques are of paramount importance. Therefore, in order to establish the PCA model from inline Raman spectra, offline analyses were first performed using HPLC and LC-MS. To gain an understanding of the impact of HME processing on the integrity of RMP within the extruded formulations, the RMP content within the extrudates was analysed by HPLC. A characteristic peak of pure RMP was observed at a retention time of 6.1 min. However, HPLC analyses of some extrudates (depending on the HME process conditions employed) also showed an additional peak, which appeared at a retention time of 7.1 min (Figure 2). As observed in figure 2, the intensity of the second peak was clearly higher for sample $\mathrm{S} 15\left(\mathrm{Temp} .=140^{\circ} \mathrm{C}\right)$ than for $\mathrm{S} 14\left(\mathrm{Temp} .=110^{\circ} \mathrm{C}\right)$, and the RMP peak decreased in intensity for S15 compared to S14, suggesting that various levels of RMP degradation were generated through HME processing. To further investigate the pathway of potential RMP degradation relating to the appearance of the UV absorption peak at a retention time of $7.1 \mathrm{~min}$ in the HPLC, LC-MS analysis was performed. The exact molecular weight may be obtained using this method for RMP before and after exposure to the heating stress induced by HME. As an example, as shown in Figure 3a, the mass spectrum of pure RMP was tested after thermal treatment at $100^{\circ} \mathrm{C}$ for two hours on a hotplate, considered to be similar to the $\mathrm{HME}$ temperature environment. It was observed that with heat treatment at $100^{\circ} \mathrm{C}$, molecular ion at m/z 417.2381 (elemental composition $\mathrm{C}_{23} \mathrm{H}_{33} \mathrm{~N}_{2} \mathrm{O}_{5}$ calculated with +/-0.8 mDa accuracy) in positive mode with $\mathrm{m} / \mathrm{z} 415.229$ (elemental composition $\mathrm{C}_{23} \mathrm{H}_{31} \mathrm{~N}_{2} \mathrm{O}_{5}$ calculated with +/- 0.4 mDa accuracy) representing the elements of unchanged RMP (C23H32N2O). Whilst, when RMP was treated at $140^{\circ} \mathrm{C}$ for two hours on a hotplate, LC-MS analyses showed different results both in positive and negative ionisation modes. The negative ionisation mode did not provide any signal which is indicative of removal of the carboxylic acid functional group after heat treatment. The trace in the positive mode ionisation showed the presence of a peak with a molecular ion at m/z 399.2291 (elemental composition $\mathrm{C}_{23} \mathrm{H}_{31} \mathrm{~N}_{2} \mathrm{O}_{4}$ calculated with +/- 0.7mDa accuracy, Figure 
3b) matching the formula of the suggested RMP degradation product (RMP-DKP) (Angeli and

251 Trezza, 2009; De diego et al., 2010; Hogan et al., 2000).

252 Previously, our study showed that RMP is thermolabile upon melting in the DSC with a first

253 degradation beginning at $123^{\circ} \mathrm{C}$ associated with a weight loss of $\sim 5 \%$ (by TGA) (Kelleher et al.,

254 2018). This percentage of RMP degradation corresponds to the loss of one $\mathrm{H}_{2} \mathrm{O}$ molecule (molar

255 mass 18) representing with the difference in molecular weights between RMP and RMP-DKP. To

256 further access the thermal degradation products post-HME processing, the extrudates were

257 analysed by both HPLC and LC-MS. Results obtained for each experiment in terms of RMP

258 content and level of RMP degradation are summarised in Table 3.

259 Table 3: Overview of the composition and process conditions performed during this study and

260 resultant HPLC data.

\begin{tabular}{|c|c|c|c|c|c|c|c|c|c|}
\hline & \multicolumn{3}{|c|}{$\begin{array}{c}\text { Formulation } \\
\text { variables }\end{array}$} & \multicolumn{3}{|c|}{ Process variables } & \multicolumn{3}{|c|}{ CQAs (Responses) } \\
\hline Ext & $\begin{array}{c}\mathbf{R M P}_{\text {theo }} \\
(\%)\end{array}$ & $\begin{array}{l}\text { TEC } \\
(\%)\end{array}$ & $\begin{array}{l}\text { EPO } \\
(\%)\end{array}$ & $\begin{array}{c}\text { Feed } \\
\text { speed } \\
(\text { rpm) }\end{array}$ & $\begin{array}{l}\text { Screw } \\
\text { speed } \\
\text { (rpm) }\end{array}$ & $\begin{array}{c}\text { Temp. } \\
\left({ }^{\circ} \mathrm{C}\right)\end{array}$ & $\begin{array}{c}\text { Drug load } \\
\text { (\%) }\end{array}$ & $\begin{array}{c}\text { Normalised } \\
\text { RMP-DKP } \\
\text { (\%)* }\end{array}$ & $\begin{array}{c}\text { Level of RMP } \\
\text { degradation } \\
(\%)\end{array}$ \\
\hline S1 & 15 & 0 & 85 & manual & 60 & 140 & $4.2 \pm 0.2$ & $7.9 \pm 0.5$ & High \\
\hline S2 & 15 & 0 & 85 & manual & 60 & 110 & $9.5 \pm 1.2$ & $4.0 \pm 0.06$ & High \\
\hline S3 & 5 & 10 & 85 & manual & 60 & 110 & $4.6 \pm 0.4$ & $0.5 \pm 0.03$ & Low \\
\hline S4 & 10 & 10 & 80 & manual & 60 & 110 & $8.9 \pm 0.3$ & $1.0 \pm 0.07$ & Low \\
\hline S5 & 15 & 10 & 75 & manual & 60 & 110 & $13 \pm 0.5$ & $1.7 \pm 0.08$ & Low \\
\hline S6 & 5 & 5 & 90 & 7 & 60 & 110 & $3.2 \pm 0.5$ & $1.2 \pm 0.03$ & Low \\
\hline S7 & 10 & 5 & 85 & 7 & 60 & 110 & $7.2 \pm 0.8$ & $2.1 \pm 0.3$ & Low \\
\hline S8 & 15 & 5 & 80 & 9 & 60 & 110 & $10.5 \pm 0.35$ & $4.8 \pm 0.3$ & High \\
\hline S9 & 5 & 5 & 90 & 2 & 5 & 110 & $5.1 \pm 0.2$ & $0.7 \pm 0.1$ & Low \\
\hline S10 & 10 & 7.5 & 82.5 & 15 & 50 & 110 & $10.45 \pm 1.3$ & $1.2 \pm 0.3$ & Low \\
\hline S11 & 10 & 7.5 & 82.5 & 15 & 50 & 110 & $10.75 \pm 0.5$ & $1.0 \pm 0.1$ & Low \\
\hline S12 & 15 & 10 & 75 & 25 & 90 & 110 & $15.2 \pm 0.35$ & $0.8 \pm 0.03$ & Low \\
\hline$S 13$ & 15 & 10 & 75 & 20 & 70 & 100 & $14.9 \pm 0.1$ & $1.0 \pm 0.3$ & Low \\
\hline$S 14$ & 15 & 10 & 75 & manual & 100 & 110 & $14.7 \pm 0.3$ & $0.2 \pm 0.2$ & Low \\
\hline S15 & 15 & 10 & 75 & manual & 100 & 140 & $10.6 \pm 0.3$ & $2.0 \pm 1.1$ & Low \\
\hline
\end{tabular}

261 * RMP-DKP level was normalised according to equation 1

262 As previously described in the methods section, the level of RMP degradation was ranked into

263 Low, Medium and High, presented in Table 3. It is evident through the use of offline analyses, 
the percentage of RMP-DKP (degradation) and differences in RMP content before and after HME processing can be quantified. These quantitative responses can be further used to probe the design space for RMP processing using HME. For example, with regard to samples S14 and S15, temperature change $\left(110-140^{\circ} \mathrm{C}\right)$ resulted in a significant decrease in RMP drug loading and a subsequent increase in the concentration of RMP-DKP.

\subsection{Offline Raman spectroscopic analysis on RAM and its degradation product}

270 To assess the feasibility of using inline Raman spectroscopic analysis to simultaneously detect 271 and quantify RMP and RMP-DKP during HME processing, it is important to check if such 272 differences between RMP and RMP-DKP (involving the loss of one $\mathrm{H}_{2} \mathrm{O}$ ) can be captured using 273 offline Raman signals. Offline Raman spectra of RMP and RMP-DKP are shown in Figure 4a. 274 Signals representing characteristic groups of the RMP molecule were observed at $1004 \mathrm{~cm}^{-1}$ 275 (aromatic ring), $1654 \mathrm{~cm}^{-1}$ (carboxylic acid) and $3278 \mathrm{~cm}^{-1}$ (secondary amine). With respect to RMP-DKP, a strong peak corresponding to the aromatic group was still observed, while the characteristic peaks of carboxylic acid and secondary amine were absent. The wavelength of the aromatic peak was chosen to characterise drug content for this study due to it being present in the RMP spectrum. Knowing that aromatic peak was not specific to RMP, a second signal region need was selected in order to characterise the part of RMP-DKP generated during HME 281 processing. Due to moderate intensity of carboxylic acid and amine groups in Raman 282 spectroscopy, wavelength regions associated with these changes were further investigated and 283 appropriate region(s) were chosen as suitable for identification of the RMP degradation product 284 through multivariate analysis study. To clarify the interferences may be contributed from other 285 excipients in the extrudates as well as the process temperature $\left(100-140^{\circ} \mathrm{C}\right)$, the inline Raman 286 spectra at the regions of interests were presented in Figure $4 \mathrm{~b}$. It is clear that, the regions 287 around $1004 \mathrm{~cm}^{-1}$ indicating of aromatic ring in RMP were not influenced. A detailed comparison 288 between RMP, triethyl citrate and Eudragit EPO at suggested regions using Raman spectroscopic analysis was provided in support document. 


\subsection{Wavelength region selections on inline Raman spectra}

291 Our main objective of this article is to identify the most important Raman spectra regions where 292 the main quality attributes (RMP content and RMP-DKP content) can be described using a 293 multivariate data analysis model. Further enrichment of this model and subsequently 294 development of Raman-based partial least square model will be conducted in our following 295 work. As previously discussed, from offline Raman spectra, Region 1 (950 to $1050 \mathrm{~cm}^{-1}$ in Figure 296 4) was selected to represent the variation of RMP content in the formulations due to the intense peak corresponding to the aromatic group in RMP. Inline Raman spectra were also collected for all the fifteen extrusion samples during HME processing. Similar features were observed from the inline Raman spectra where Region 1 data collected for all HME experiments are presented

300 in Figure 5. To decrease the additional noise during inline Raman acquisition, pre-processing 301 filters (SNV+MSC+SG) were applied to the spectra. As observed in Figure 5a, the intensity of the 302 peak at $1004 \mathrm{~cm}^{-1}$ is linked to RMP concentration. The loading plot (Figure $5 \mathrm{~b}$ ) confirmed the 303 significance of this wavelength region in relation to the drug loading. In this case, the aromatic 304 peak has the largest absolute value in the loading plot (p1) justifying the importance of this 305 variable in building the PCA scatter plot. Thus, a PC 1 versus PC 2 scatter plot for the region 1 in 306 Raman spectra was obtained (Figure 6). The correct selection of the wavelength region on the 307 Raman spectra resulted in the main scores being oriented along PC $1\left(\mathrm{R}^{2}=94 \%\right)$. The spectra 308 having the highest PC 1 score correspond to the extrudates containing $15 \% \mathrm{w} / \mathrm{w}$ of RMP 309 (orange) while a low value of PC 1 was associated with RMP concentration of 5\% w/w (blue). However, some samples containing 15\% w/w RMP (theoretical content) were present with 311 medium scores on PC 1, indicating the complexity of developing a PCA model using single region 312 data. The discrepancy between high and low RMP drug loading when using Region 1 alone may 313 be attributed to the corresponding Raman spectra region characteristic of benzene ring group.

314 The benzene ring structure is present in both RMP and RMP-DKP, and thus various levels of 315 RMP-DKP present in the extrudates under different process conditions will affect the accuracy of 316 the PCA model when only Region 1 was used. Thus, the PCA model based on wavelength range 
317 Region 1 alone was not sufficient to describe the mixture of RMP and RMP-DKP within the 318 extrudates.

319 After further investigation, Region 2 (1150 to $1250 \mathrm{~cm}^{-1}$ ) in the Raman spectra collected from 320 inline Raman signals was selected and filters were applied to highlight the level of RMP 321 degradation, i.e. \%RMP-DKP in the extrudates as quantified by HPLC (Figure 7). Spectral regions 322 associated with the carboxylic acid and secondary amines were not significant in the PCA models 323 and hence were neglected from consideration. Spectra from Region 2 have also shown 324 significant variations in responding to RMP degradation, a high amplitude in Raman signal was 325 observed for RMP degradation at low level, whilst a significant decrease in the amplitude was 326 observed with increased level of RMP degradation (\%RMP-DKP). The loading plot (Figure 7b) 327 confirms that peaks selected between $1150 \mathrm{~cm}^{-1}$ and $1250 \mathrm{~cm}^{-1}$, are indeed responsive variables. 328 This can also be correlated with offline measurement of pure RMP-DKP samples showing 329 broadened peaks with lower amplitude (Figure 4). Across the different degradation levels, a 330 correlation of $72.3 \%$ was obtained along the PC 1 (Region 2) (Figure 8). A high level of 331 degradation ( $>4 \%$ ) was observed on the negative side of PC 1 while a low-level degradation was 332 observed on the positive side. When PC $2\left(\mathrm{R}^{2}=23 \%\right)$ was considered, RMP drug content 333 (RMP theo) can also be identified (labelled at 5\%, 10\% and 15\%). Furthermore, clusters

334 corresponding to 5\% RMP content were observed in the negative values of PC 2, clusters 335 representing $10 \%$ of drug content were localised close to zero and groups corresponding to $15 \%$ 336 of RMP were found in the positive values of PC 2 . These indicate that Region 2 can also be used 337 to describe the level of RMP degradation as well as RMP drug content.

\subsection{Establishment of PCA model from inline Raman spectra}

339 Finally, when RMP drug content (950-1050 $\mathrm{cm}^{-1}$, Region 1) and RMP degradation (1150-1250

$340 \mathrm{~cm}^{-1}$, Region 2) regions were combined, a PCA model was established based on chosen 420 inline 341 Raman spectra (Figure 9). PC 1 with $\mathrm{R}^{2}$ of $50.1 \%$ and PC 2 with $\mathrm{R}^{2}$ of $45 \%$ were obtained, 342 indicating a good identification for both RMP drug content and its degradation within 
extrudates. The level of RMP-DKP was oriented along PC 1 scores, while pure RMP content was

344 oriented along PC 2 scores. With this PCA model obtained through inline Raman spectra, we 345 were able to characterise CQAs (RMP drug content and \%RMP-DKP level) for our systems during 346 HME processing. The RMP drug content and its degradation product were separated along two 347 main principal components in this PCA model. Combining observations of RMP and its 348 degradation product with results obtained from offline HPLC analysis (Table 1), maps were 349 established to summarise the CQAs based on the chosen responses (Figure $10 \mathrm{a} \& \mathrm{~b}$ ). Regarding 350 degradation, RMP-DKP (PC 1 scores alone), three levels of degradation were identified, from 351 low, medium and high (Figure 10a).

352 More importantly, we were also able to simultaneously identify the level of RMP drug content 353 within the extrudates during HME processing.

\subsection{Probing the effects of process parameters on the CQAs}

355 In the past decade, the pharmaceutical and biotechnological industry have developed integrated 356 "one input and one output" powder to tablet production lines. The possibility of inline quality 357 assessment of CQAs and real-time quality control will provide the necessary framework for the 358 future of pharmaceutical manufacturing (Fonteyne et al., 2015). In this work, RMP content and 359 the level of \%RMP-DKP were considered as two of the most important quality attributes of the 360 prepared extrudates. The development of a PCA model was based on highlighting these CQAs 361 from an inline Raman-based multivariate data analysis technique. As discussed, CQAs might be 362 influenced by material attributes (CMAs) and process parameters (CPPs). In this scoping design, 363 plasticizer TEC concentration, barrel temperature, feed speed and screw rotation speed were all 364 changed. The influence of these investigated parameters on the CQAs were assessed in situ using 365 inline Raman spectroscopy, based upon our established PCA map.

366 Although this scoping design was not a full experimental design normally used to probe the 367 critical factors on the CQAs, the inline Raman-based PCA mapped using the existing dataset 368 revealed important aspects for the current study. For example, the effects of TEC on the level of 
RMP degradation has been highlighted in Figure 10b. Along PC 1, extrudates with a high level of TEC were observed with low level of RMP degradation (positive scores in PC 1) and samples

371 without TEC were observed with high level of RMP degradation (negatively scored in PC 1). As

372 previously discussed, PC 1 is associated with the level of RMP degradation, this observation 373 allows showing positive effect of TEC on RMP integrity with a degradation decreases when TEC 374 increases. Additionally, when samples prepared with a TEC concentration of $10 \%$ (blue clusters 375 in Figure 10b) were considered, variations can also be observed throughout PC 1, suggesting the influence of other parameters on the level of RMP degradation. For example, formulation trials S1 and S15 were both prepared at $140^{\circ} \mathrm{C}$. S1 was negatively scored along PC 1, while S15 was localised at zero, implying high and medium RMP degradation (RMP-DKP), respectively. The

379 addition of TEC and increase in screw speed in S15 results in a decrease in degradation, even 380 though the process temperature was set at $140^{\circ} \mathrm{C}$.

381 Results therefore suggest that the level of TEC, feed speed and screw rotating speed are all 382 contributed to the level of RMP degradation produced via HME. Recently, the contributions of 383 HME process parameters to the specific mechanical energy inputs and residence time of the 384 process have also been highlighted in the literature (Repka et al., 2018). Plasticizer lowers the 385 glass transition temperature $\left(\mathrm{T}_{\mathrm{g}}\right)$ as well as the melt viscosity of the polymer, resulting in a 386 reduction in the shear-induced temperature changes (Chokshi and Zia, 2004). Therefore, 387 inclusion of plasticizer decreases the heat stress experienced during HME processing, hence 388 reducing the generation of RMP-DKP. The factors that influence the discrepancy between the 389 extruder temperature and local material temperature within the extruder are pertinent to 390 understanding and controlling this platform for the production of thermolabile drugs (Huang et 391 al., 2017).

392 Furthermore, the effect of heat stress on the degradation of RMP is well known in the literature

393 (Figure 3). The PCA scatterplot was also used to identify the influence of processing 394 temperature, as shown in Figure 11. As predicted using offline measurements, when a 
temperature of $100^{\circ} \mathrm{C}$ was used during HME processing, the level of RMP degradation was not

396 observed/measurable, whilst when a high temperature $\left(140^{\circ} \mathrm{C}\right)$, was used for $\mathrm{HME}$ processing,

397 medium to high levels of RMP degradation were observed. In order to highlight the potential 398 temperature effect on both CQAs during processing, extrudates were prepared at different 399 temperatures $\left(100^{\circ} \mathrm{C}\right.$ for $\mathrm{S} 14$ and $140^{\circ} \mathrm{C}$ for S15) may be compared. These extrudates were 400 obtained under the same conditions, except for processing temperature (S14 at $110^{\circ} \mathrm{C}$ and S15 401 at $140^{\circ} \mathrm{C}$ ). S14 resulted in positive scoring of PC 1, while S15 was localised in the centre of the 402 scatterplot, implying a lower \%RMP-DKP level for S14 compared to S15. This result was also confirmed by HPLC analysis with $0.2 \%$ and $2 \%$ of RMP-DKP obtained for S14 and S15 respectively (Table 1 ).

However, when a medium temperature of $110^{\circ} \mathrm{C}$ was applied during HME processing, various levels of RMP degradation were obtained (highlighted as blue, Figure 11). The effect of TEC content, for example, at the same processing temperature can be demonstrated by a comparison between S2 and S14 formulations, which were both produced at a temperature of $110^{\circ} \mathrm{C}$. A $4 \%$ difference in RMP degradation was observed between the two samples, simply due to the TEC loading (0\% w/w TEC in S2 extrudates versus 10\% w/w TEC in S14 extrudates). Additionally, 411 the effect of initial drug loading may also play an important role on the rate of degradation; in 412 comparing formulations with different initial drug loads (S8 and S9 formulations). For the S8 413 formulation (15\% RMP, processed at $110^{\circ} \mathrm{C}$ with $5 \%$ TEC and 60 rpm screw speed), 4.8\% RMP414 DKP was detected in the final product, whilst for the S9 formulation (5\% RMP that was 415 processed at similar conditions), only 0.7\% RMP-DKP was observed. This suggests that, with 416 increasing RMP drug loading, there is an increased chance of RMP exposure to heating and 417 shearing stresses, which may encourage the generation of the RMP-DKP degradation product. 418 Therefore, the process conditions are highly sensitive to the initial RMP drug loading. Cares have 419 to be taken in consideration of the design space for HME platform at different drug loadings to 420 ensure for efficient production of thermally liable drugs. 
421 In general, the PAT tools equipped with multivariate data analysis reduces process errors and 422 provide a quality product (Challa and Potumarthi, 2013). Through the investigation of these 423 critical factors using an established PCA scatterplot and offline RMP/RMP-DKP results, we could 424 directly identify, in principle, the main route causes for RMP degradation within extruded 425 formulations, during HME processing. Furthermore, results also indicate that the TEC content 426 and process temperature are not the only parameters influencing the generation of RMP-DKP 427 during HME (Figure 10 and 11). Further investigations of the formulation design in relation to 428 the HME process parameters will be conducted using a full design of experiments and PLS model. The design space for RMP-HTCZ based fixed-dose formulations will be assessed in more detail through the inline Raman-PLS tool.

\subsection{Inline Raman based partial least square model}

432 Although, the main objectives of this article was to test the feasibility of inline Raman based 433 PCA model for the identification of RMP and RMP-DKP within the hot-melt extrusion 434 process, with the offline HPLC quantification on the RMP content, a quantitative model based on partial least square method (PLS) may also be constructed. The establishment of inline

436 Raman-based PLS model can help us to further test the suitability of the selected Raman 437 regions $\left(950-1250 \mathrm{~cm}^{-1}\right)$ in the PCA model. Figure 12 shows the constructed PLS model 438 based on the inline Raman spectra collected from extrusion trials and the RMP drug loadings 439 quantified by HPLC (Table 3). A goodness fitting $\left(\mathrm{R}^{2}\right)$ of 0.9427 has been obtained with a 440 root mean square error of estimation (RMSEE) value of 1.05 and root mean square error of 441 internal cross validation (RMSEcV) value of 1.12. Clearly, external validations are required to 442 further develop the quantitative model, nevertheless, the values of RMSEE and RMSEcV 443 indicate a good level of predictively for RMP content and validate the selection of the regions 444 from inline Raman spectra.

\section{Conclusions}


446 The application of HME as a means of continuously manufacturing thermally liable drugs has

447 been limited due to the degradation caused during processing. In this article, we have 448 demonstrated the use of a Raman-based process analytical tool to probe the effects of 449 formulation design and process parameters on the degradation of thermally liable drug RMP. 450 Inline monitoring and characterisation were performed using Raman spectroscopy and 451 meaningful conclusions were drawn via the establishment of a multivariate data analysis model. 452 With this model, it was possible to convert the complex Raman data into a principle component 453 map, where the levels of RMP drug content and RMP-DKP degradation were successfully 454 mapped into clusters. Two specific Raman regions were firstly selected to characterise RMP 455 content (Region 1) and its degradation product (Region 2). Through the combination of both 456 regions $\left(950 \mathrm{~cm}^{-1}\right.$ to $\left.1250 \mathrm{~cm}^{-1}\right)$, the PCA scatterplot obtained correlated to the level of RMP-DKP 457 along principle component one $\left(\mathrm{R}^{2}=50.1 \%\right)$ and RMP content along principle component two $458\left(\mathrm{R}^{2}=45 \%\right)$. This map could differentiate between the level of RMP-DKP and RMP drug content, 459 which can also be related to the critical formulation design and process parameters for the HME 460 processing. Data analysis of the PCA scatterplot highlighted the impact of TEC concentration and 461 process temperature on RMP degradation within the extruded formulations. The establishment 462 of Raman-based PCA map will be further utilised for the optimisation of HME processing to 463 produce RMP-HCTZ fixed-dose combination formulations. 
466 This investigation was conducted with the financial support of both the Department for 467 the Economy of Northern Ireland and Science Foundation Ireland (SFI) under Grant 468 Number 14/IA/2559.

469 


\section{References}

Angeli, D.G., Trezza, C., 2009. Quality and stability of ramipril generics/copies versus reference ramipril (Tritace®): A 3-month stability comparative study. Clin. Drug Investig. 29, 667676. https://doi.org/10.2165/11315270-000000000-00000

Badman, C., Trout, B.L., 2014. Achieving Continuous Manufacturing. Int. Symp. Contin. Manuf. Pharm. 104, Intro White Paper. https://doi.org/10.1002/jps.24246

Baronsky-Probst, J., Möltgen, C. V., Kessler, W., Kessler, R.W., 2016. Process design and control of a twin screw hot melt extrusion for continuous pharmaceutical tamper-resistant tablet production. Eur. J. Pharm. Sci. 87, 14-21. https://doi.org/10.1016/j.ejps.2015.09.010

Challa, S., Potumarthi, R., 2013. Chemometrics-based process analytical technology (PAT) tools: Applications and adaptation in pharmaceutical and biopharmaceutical industries. Appl. Biochem. Biotechnol. 169, 66-76. https://doi.org/10.1007/s12010-012-9950-y

Chokshi, R., Zia, H., 2004. Hot Melt Extrusion Technique: A Review. Iran. J. Pharm. Res. 3-16.

De Beer, T., Burggraeve, A., Fonteyne, M., Saerens, L., Remon, J.P., Vervaet, C., 2011. Near infrared and Raman spectroscopy for the in-process monitoring of pharmaceutical production processes. Int. J. Pharm. 417, 32-47. https://doi.org/10.1016/j.ijpharm.2010.12.012

De diego, M., Godoy, G., Olivares, M., Mennickent, S., Godoy, R., 2010. Stress degradation studies of ramipril by a validated stability-indicating liquid chromatographic method. J. Chil.Chem.

Eitzlmayr, A., Koscher, G., Reynolds, G., Huang, Z., Booth, J., Shering, P., Khinast, J., 2014. Mechanistic modeling of modular co-rotating twin-screw extruders. Int. J. Pharm. 474, 157176. https://doi.org/10.1016/j.ijpharm.2014.08.005

European Medicines Agency, 2015. ICH guideline Q8 ( R2 ) on pharmaceutical development. Ema/Chmp/Ich/167068/2004 8, 16706. https://doi.org/EMEA/CHMP/167068/2004 ICH 
FDA, 2004. Guidance for Industry Guidance for Industry PAT - A Framework for Innovative Pharmaceutical Development, Manufacturing, and Quality Assurance.

Fonteyne, M., Soares, S., Vercruysse, J., Peeters, E., Burggraeve, A., Vervaet, C., Remon, J.P., Sandler, N., De Beer, T., 2012. Prediction of quality attributes of continuously produced granules using complementary pat tools. Eur. J. Pharm. Biopharm. 82, 429-436. https://doi.org/10.1016/j.ejpb.2012.07.017

Fonteyne, M., Vercruysse, J., De Leersnyder, F., Van Snick, B., Vervaet, C., Remon, J.P., De Beer, T., 2015. Process Analytical Technology for continuous manufacturing of solid-dosage forms. TrAC Trends Anal. Chem. 67, 159-166. https://doi.org/10.1016/j.trac.2015.01.011

Hanyšová, L., Václavková, M., Dohnal, J., Klimeš, J., 2005. Stability of ramipril in the solvents of different pH. J. Pharm. Biomed. Anal. 37, 1179-1183. https://doi.org/10.1016/j.jpba.2004.10.041

Hogan, B.L., Williams, M., Idiculla, A., Veysoglu, T., Parente, E., 2000. Development and validation of a liquid chromatographic method for the determination of the related substances of ramipril in Altace capsules. J. Pharm. Biomed. Anal. 23, 637-651. https://doi.org/10.1016/S0731-7085(00)00342-3

Huang, S., O’Donnell, K.P., Delpon de Vaux, S.M., O’Brien, J., Stutzman, J., Williams, R.O., 2017. Processing thermally labile drugs by hot-melt extrusion: The lesson with gliclazide. Eur. J. Pharm. Biopharm. 119, 56-67. https://doi.org/10.1016/j.ejpb.2017.05.014

Karandikar, H., Ambardekar, R., Kelly, A., Gough, T., Paradkar, A., 2015. Systematic identification of thermal degradation products of HPMCP during hot melt extrusion process. Int. J. Pharm. 486, 252-258. https://doi.org/10.1016/j.ijpharm.2015.04.007

Kelleher, J.F., Gilvary, G.C., Madi, A.M., Jones, D.S., Li, S., Tian, Y., Almajaan, A., Senta-Loys, Z., Andrews, G.P., Healy, A.M., 2018. A Comparative Study between Hot-Melt Extrusion and 
Spray-Drying for the Manufacture of Anti-Hypertension Compatible Monolithic Fixed-Dose

$\begin{array}{lllll}\text { Combination } & \text { Products. Int. J. Pharm. 545, 183- }\end{array}$ https://doi.org/10.1016/j.ijpharm.2018.05.008

Kelly, A.L., Gough, T., Dhumal, R.S., Halsey, S.A., Paradkar, A., 2012. Monitoring ibuprofennicotinamide cocrystal formation during solvent free continuous cocrystallization (SFCC) using near infrared spectroscopy as a PAT tool. Int. J. Pharm. 426, 15-20. https://doi.org/10.1016/j.ijpharm.2011.12.033

Lang, B., McGinity, J.W., Williams, R.O., 2014. Hot-melt extrusion - basic principles and pharmaceutical applications. Drug Dev. Ind. Pharm. 40, 1133-1155. https://doi.org/10.3109/03639045.2013.838577

Laske, S., Paudel, A., Scheibelhofer, O., Sacher, S., Hoermann, T., Khinast, J., Kelly, A., Rantannen, J., Korhonen, O., Stauffer, F., De Leersnyder, F., De Beer, T., Mantanus, J., Chavez, P.F., Thoorens, B., Ghiotti, P., Schubert, M., Tajarobi, P., Haeffler, G., Lakio, S., Fransson, M., Sparen, A., Abrahmsen-Alami, S., Folestad, S., Funke, A., Backx, I., Kavsek, B., Kjell, F., Michaelis, M., Page, T., Palmer, J., Schaepman, A., Sekulic, S., Hammond, S., Braun, B., Colegrove, B., 2017. A Review of PAT Strategies in Secondary Solid Oral Dosage Manufacturing of Small Molecules. J. Pharm. Sci. 106, 667-712. https://doi.org/10.1016/j.xphs.2016.11.011

Lee, S.., 2015. Modernizing Pharmaceutical Manufacturing: from Batch to Continuous Production. J. Pharm. Innov. 10, 191-199.

Li, S., Yu, T., Tian, Y., McCoy, C.P., Jones, D.S., Andrews, G.P., 2016. Mechanochemical synthesis of pharmaceutical cocrystal suspensions via hot melt extrusion: Feasibility studies and physicochemical characterization. Mol. Pharm. 13, 3054-3068. https://doi.org/10.1021/acs.molpharmaceut.6b00134

542 Netchacovitch, L., Thiry, J., De Bleye, C., Chavez, P.F., Krier, F., Sacré, P.Y., Evrard, B., Hubert, P., 
Ziemons, E., 2015. Vibrational spectroscopy and microspectroscopy analyzing qualitatively and quantitatively pharmaceutical hot melt extrudates. J. Pharm. Biomed. Anal. 113, 21-33.

Paradkar, A., Dhumal, R.S., Kelly, A.L., York, P., Coates, P.D., 2010. Cocrystalization and https://doi.org/10.1016/j.jpba.2015.01.051

Patil, H., Tiwari, R. V., Repka, M.A., 2016. Hot-Melt Extrusion: from Theory to Application in Pharmaceutical Formulation. AAPS PharmSciTech 17, $\quad 20-42$. https://doi.org/10.1208/s12249-015-0360-7

552 553

Rantanen, J., Khinast, J., 2015. The Future of Pharmaceutical Manufacturing Sciences. J. Pharm. Sci. 104, 3612-3638. https://doi.org/10.1002/jps.24594

Repka, M. a, Battu, S.K., Upadhye, S.B., Thumma, S., Crowley, M.M., Zhang, F., Martin, C., McGinity, J.W., 2007. Pharmaceutical applications of hot-melt extrusion: Part II. Drug Dev. Ind. Pharm. 33, 1043-1057. https://doi.org/10.1080/03639040701525627

Repka, M.A., Bandari, S., Kallakunta, V.R., Vo, A.Q., McFall, H., Pimparade, M.B., Bhagurkar, A.M., 2018. Melt extrusion with poorly soluble drugs - An integrated review. Int. J. Pharm. 535, 68-85. https://doi.org/10.1016/j.ijpharm.2017.10.056

Tian, Y., Jones, D.S.D.S., Donnelly, C., Brannigan, T., Li, S., Andrews, G.P.G.P., 2018. A New Method of Constructing a Drug-Polymer Temperature-Composition Phase Diagram Using Hot-Melt Extrusion. Mol. Pharm. 15 1379-1391. https://doi.org/10.1021/acs.molpharmaceut.7b00445

Wahl, P.R., Treffer, D., Mohr, S., Roblegg, E., Koscher, G., Khinast, J.G., 2013. Inline monitoring and a PAT strategy for pharmaceutical hot melt extrusion. Int. J. Pharm. 455, 159-168. https://doi.org/10.1016/j.ijpharm.2013.07.044 
567 ZION market research, 2018. Global Pharmaceutical Continuous Manufacturing Market Report.

568 